\title{
Estudio fotoquímico del Liquen everniopsis trulla y la determinación de la actividad antioxidante
}

Phytochemical study of Lichen everniopsis trulla and determination of antioxidant activity

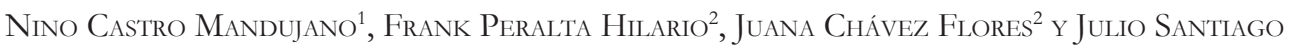 \\ CONTRERAS $^{1}$
}

\section{RESUMEN}

En esta investigación se ha realizado el estudio fitoquímico del liquen Everniopsis trulla. Se realizó la extracción a $400 \mathrm{~g}$ de muestra seca y molida con etanol, luego, se fraccionó y purificó aplicando los métodos de cromatografía en columna, solubilidad y recristalización, empleando diferentes solventes; finalmente se obtuvo unos cristales amarillos (sólido A, ácido úsnico) de punto de fusión de $203^{\circ} \mathrm{C}$, el cual se elucidó su estructura en base a los datos espectroscópicos (UV-Visible, IR, RMN-H1, RMN-C13 y espectrometría de masas). Además, se realizó determinación de metales, y determinaciones de las actividades antiinflamatorias y antioxidantes del compuesto aislado. Finalmente se determinó el efecto antiinflamatorio del ácido úsnico, se determinó por el modelo in vitro de edema inducido por xilol en la oreja derecha del ratón, la crema 0,5\% elaborada a partir del sólido A y del extracto etanólico; los cuales mostraron 37,72\% 19,54\% de inhibición en la oreja derecha del ratón. También se realizó el análisis de fenoles totales por el método de DPPH el extracto del liquen, el cual dio 206 mg de ácido gálico por gramo de extracto. Además se logró determinar la actividad antioxidante en el extracto del liquen con mayor contenido de compuestos fenólicos, mediante el empleo del reactivo DPPH. Se obtuvo un valor de CI50 a una concentración de $100 \mathrm{mg} / \mathrm{mL}$.

Palabras clave: actividad antiinflamatoria; antioxidante; everniopsis trulla; ácido úsnico.

\footnotetext{
1 Universidad Nacional Mayor de San Marcos. Lima, Perú.

2 Universidad Privada Norbert Wiener. Lima, Perú.
} 


\section{ABSTRACTS}

In this research, it has been carried out phytochemical study of lichen Everniopsis trulla. Extraction $400 \mathrm{~g}$ dry sample was ground with ethanol and then fractionated using methods and purified by column chromatography, recrystallization and solubility using different solvents; finally, was obtained solid A (usnic acid, yellow crystals of melting point $203^{\circ} \mathrm{C}$ ), which its structure was elucidated by spectroscopic data (UV-visible, IR, 1H-NMR, 13C-NMR and mass spectrometry). In addition, determination of metals was carried out, and anti-inflammatory activity determinations and antioxidants of 3 samples (lichen extract, solid A and B).

Finally, the anti-inflammatory effect of the extract, solid A and solid B was determined, it was determined by the in vitro model of xylene induced edema in the right ear of mouse, $0.5 \%$ cream made from, ethanol extract, the solid A and B; which showed $42.21 \%, 37.72 \%$ and $38.32 \%$ inhibition on the right ear of the mouse. Total phenols analysis was also performed by the method of DPPH the lichen extract, which gave $206 \mathrm{mg}$ of gallic acid per gram of extract. In addition it was determined the antioxidant activity in the lichen extract more phenolic content, by using DPPH reagent. An IC50 at a concentration of $100 \mathrm{mg} / \mathrm{mL}$ was obtained.

Keywords: anti-inflammatory activity; antioxidant; Everniopsis trulla; ácido úsnico.

\section{INTRODUCCIÓN}

El líquen es un organismo simbiótico conformado por un hongo y una alga. Los líquenes, producen metabolitos secundarios que son compuestos fenólicos, propios de ellos, los cuales se acumulan en talos del líquen (dépsidos, depsidonas, dibenzofuranos y ácidos úsnicos), (Kika, 1998).

Los compuestos liquénicos son bio-indicadores de la contaminación ambiental, tienen propiedades farmacológicas, son antioxidantes, en Japón y China se consumen en ensaladas, etc. (Huneck, 1996).

Por ello, fue nuestro interés el de realizar un estudio fitoquímico de la Everniopsis trulla, para ver sus propiedades antioxidantes y otros componentes que nos permitan asegura que es posible y recomendable consumir en ensaladas; también analizar sus antinflamatorias, ya que esta especie se viene consumiendo contra la tos y otras enfermedades respiratorias en los lugares que hemos recolectado. 


\section{MATERIALES Y MÉTODOS}

Recolección y secado de la muestra, la planta liquénica completa se colectó en mayo de 2013 en el departamento de Áncash, provincia de Asunción, distrito de Chacas, pueblo de Canchas; a una altitud de $3427 \mathrm{msnm}$. El liquen Everniopsis trulla (fue identificada por la Dra. Magda Chanco, del Museo de Historia Natural - UNMSM), esta muestra se secó en la estufa (Memmert ${ }^{\circledR}$ ) a $40{ }^{\circ} \mathrm{C}$. Durante 5 días.

Marcha fitoquímica, en la bibliografía se encuentran diferentes métodos para la detección preliminar de los diversos constituyentes químicos en la planta, llamado marcha fitoquímica o screening fitoquímico (Lock, 1994); también se ha desarrollado diversas pruebas de coloración y/o precipitación, es decir, se ha realizado pruebas especificas para identificar compuestos liquénicos (Huneck, 1996).

Análisis de metales.- El método aplicado para la muestra de liquen es una modificación del método la EPA 200.7, Rev. 4.4. EMMC, 1994; en primer lugar se realizó una digestión de la muestra seca, para ello se llevó a cenizas, calentando en la estufa a $600{ }^{\circ} \mathrm{C}$, por 5 horas; luego a las cenizas se las atacó con ácidos concentrados $(10 \mathrm{~mL}$ de $\mathrm{HCl}$ y $\mathrm{HNO} 3)$ y finalmente se llevó a una solución ácida $500 \mathrm{~mL}$. Posteriormente se llevó al equipo ICP (EPA, 1994).

La determinación de fenoles, se realizó mediante la técnica de Folin-Ciocalteau, la cual se basa en la propiedad de los compuestos fenólicos de reaccionar frente a agentes oxidantes. Este reactivo contiene molibdato y tungstato sódico que al reaccionar con los compuestos fenólicos presentes, forman complejos fosfomolíbdico - fosfotúngstico. En medio básico la transferencia de electrones reduce estos complejos a óxidos de tungsteno $\left(\mathrm{W}_{8} \mathrm{O}_{23}\right)$ y molibdeno $\left(\mathrm{Mo}_{8} \mathrm{O}_{23}\right)$, cromógenos de color azul intenso que son proporcionales a la cantidad de grupos fenólicos presentes en la molécula de interés. La lectura de la absorbancia del complejo se realizó a $760 \mathrm{~nm}$ en un espectrómetro ultravioleta-visible. Se realizó una curva de calibración con ácido gálico (patrón). Los resultados fueron expresados en $\mathrm{mg}$ de ácido gálico equivalentes por gramo de extracto de liquen (Manojlovic, 2012). Solución patrón: ácido gálico 0,1g/L Solución de carbonato de sodio al 20\% (w/v). Reactivo Folin-Ciocalteau 1N.

Preparación de la muestra, se realiza un extracto del liquen a 5 gramos con 50 $\mathrm{mL}$ del sistema $\mathrm{MeOH}-\mathrm{H}_{2} \mathrm{O}$ (1:1), empleando un ultrasonido por 10 minutos a $40^{\circ} \mathrm{C}$. se concentra y se denomina muestra M1a este extracto. El solvente empleado para todas las diluciones es el sistema $\mathrm{MeOH}_{-} \mathrm{H}_{2} \mathrm{O}$ (1:1).

Preparación de curva de calibración, a partir de la solución de ácido gálico de $100 \mathrm{mg} / \mathrm{L}$, se preparan una serie de diluciones con agua destilada para obtener $1,2,3,4,5 \mathrm{mg} / \mathrm{L}$ adicionalmente se añadió a cada vial $100 \mu \mathrm{L}$ del reactivo de 
Folin-Ciocalteau $1 \mathrm{~N}$ se agitó y luego se agregó $300 \mu \mathrm{L}$ de carbonato de sodio al $20 \%$. Y se completa con el sistema $\mathrm{MeOH}-\mathrm{H}_{2} \mathrm{O}$ a un volumen final de 2 $\mathrm{mL}$. Finalmente, se tomó la lectura en el espectrofotómetro UV a $760 \mathrm{~nm}$. El blanco tuvo los mismos componentes excepto el ácido gálico (Cruzado, 2013).

Para la determinación de fenoles, se pesó 3,6 mg de M1, el cual se disolvió en $50 \mathrm{~mL}$ de agua destilada. Se tomó $0,5 \mathrm{~mL}$ de la disolución, se le añadió $500 \mu \mathrm{L}$ del reactivo de Folin-Ciocalteau 1 N. Se dejó reposar alrededor de 5 minutos y se adicionó $300 \mu \mathrm{L}$ de carbonato de sodio al $20 \%$. Se agitó y se dejó reposar por $2 \mathrm{~h}$. Se analizó por UV-V a $760 \mathrm{~nm}$ (Cruzado, 2013).

Determinación de la actividad Antioxidante, el radical DPPH posee un electrón desapareado, lo cual, le confiere un color violeta observado en la respectiva banda de absorción alrededor de los $517 \mathrm{~nm}$. El parámetro que se midió es el porcentaje de reducción del DPPH (Q) frente a la muestra, llamado también de inhibición Se define Q con la siguiente ecuación:

$$
\mathrm{Q}=100\left(1-\left(\mathrm{A}_{\text {muestra }} / \mathrm{A}_{\text {control }}\right)\right)
$$

Donde: $\mathrm{A}_{\text {muestra }}=$ absorbancia de la muestra y $\mathrm{A}_{\text {control }}=$ absorbancia del reactivo $\mathrm{DPPH}$

Materiales y reactivos, la solución de DPPH de $150 \mu \mathrm{mol} / \mathrm{L}$ en metanol/H2O 1:1. Esta solución fue preparada antes de cada uso. Solución patrón: se realizó un control positivo con ácido gálico, para lo cual se preparó las diluciones del ácido 1, 5, 10, 15, 20, 25, 30, 35, $40 \mathrm{mg} / \mathrm{mL}$.

Preparación de la solución muestra problema: se disolvió $5 \mathrm{~g}$ de la muestra en 50 $\mathrm{mL}$ de $\mathrm{MeOH}-\mathrm{H}_{2} \mathrm{O}$ 1:1. La solución se filtró, se concentró hasta sequedad (M1, $1250 \mathrm{mg}$ de extracto) y se preparó soluciones diluidas de 5, 25, 50, 75, 100, 125, 150, 175, $200 \mathrm{mg} / \mathrm{mL}$ de muestra.

Determinación de la actividad antioxidante, se tomó $250 \mu \mathrm{L}$ de cada dilución de M1 en viales de $10 \mathrm{~mL}$ protegidos de la luz, se adicionó $1750 \mu \mathrm{L}$ de la solución de DPPH. Se agitó y se dejó en reposo durante 30 minutos. Finalmente, se leyó en el espectrofotómetro UV - V a $517 \mathrm{~nm}$. Se realizó el mismo procedimiento para la solución patrón de ácido gálico usado como estándar. Finalmente, se determinó el porcentaje de reducción del DPPH (Q), tanto para M1, como para las diluciones de ácido gálico (Cruzado,2013 y Manojlovic, 2012).

Análisis cromatográfico del extracto de la E. trulla, en esta parte primero se aplicó un método conocido el cual comprende 2 etapas, en la primera se realiza la extracción exhaustivamente de los ácidos grasos con $\mathrm{MeOH}_{-} \mathrm{CHCl}_{3} 1: 1$ y la segunda, comprende la saponificación, hidrólisis ácida y metilación de los ácidos grasos seguida del análisis por CG, (Dembisatsky 1992). 
Por otro lado, se empleó otro método, el cual consiste en: a $200 \mathrm{mg}$ de muestra de extracto $\mathrm{MeOH}-\mathrm{CHCl}_{3}$, se añadió $2 \mathrm{~mL}$ de $\mathrm{KOH}$, se colocó por 10 minutos en el ultrasonido, luego se añade hexano $2 \mathrm{~mL}$ y 10 minutos más en el ultrasonido, finalmente se centrifuga y se recogió la parte orgánica; para inyectar luego en el equipo. Los análisis se realizaron en un cromatógrafo de gases perteneciente al Laboratorio de Análisis y Monitoreo Ambiental, Servicios Analíticos Generales S.A.C. (Lima, Perú).

Aislamiento del ácido úsnico.- El liquen seco se pulverizó, usando el molino de cuchillas Willey Hill St. Model $\mathrm{N}^{\circ} 3$, obteniéndose un polvo fino. Se pesó $400 \mathrm{~g}$ de polvo y se realizó la extracción por maceración con etanol de $96^{\circ}$ a temperatura ambiente, por 4 días y se repite dos veces más, de la solución obtenida unos 3 litros, la mitad del macerado se concentró en el rotavapor (Buchi $\mathrm{R}$-210) solo hasta $200 \mathrm{~mL}$ y se llevó a refrigerar por un día a $0^{\circ} \mathrm{C}$, aquí se observó la formación de solidos amarillos, los cuales se filtraron y se recristalizaron con el sistema $\mathrm{CHCl}_{3}-\mathrm{MeOH}(1: 1 \mathrm{v} / \mathrm{v})$ para purificarlos y finalmente se obtuvo unos cristales amarillos, de punto de fusión de $203^{\circ} \mathrm{C}$, estos cristales se enviaron al extranjero para su análisis espectroscópicos.

Análisis antiinflamatorio del sólido A, se utilizó el modelo de edema auricular (Gonzales, 2007 y Seydel, 2002). El cual, consiste en la inducción de inflamación por aplicación de xilol, principal responsable de la acción irritante, en el pabellón de la oreja del ratón, la evaluación de la técnica está dada por la medición de la respuesta inflamatoria que se traduce por el aumento de peso que se produce en el área lesionada.

Para la evaluación de la actividad antiinflamatoria se utilizaron 49 ratones cepa Balb/C53/CNPB Mus músculus provenientes del Instituto Nacional de Salud de Productos Biológicos, de ambos sexos, distribuidos al azar en 7 grupos. Los ratones recibieron en la oreja derecha e izquierda el agente irritante en la cara externa e interna con un hisopo estéril con pequeña cantidad de xilol frotando por cinco veces cada cara menos el grupo control (crema base). Aplicada la sustancia flogógena se dejó trascurrir 20 minutos luego se procedió aplicar por vía tópica la crema elaborada con el sólido A (ácido úsnico) en diferentes concentraciones $(5,10,15$ y 20\%), se usó como estándar: diclofenaco $1 \%$ gel y betametasona $0,05 \%$ crema. Transcurridas 4 horas se sacrificaron al animal de experimentación (ratones) por dislocación cervical y luego se procede a cortar una porción de ambas orejas utilizando un sacabocados ( $6 \mathrm{~mm}$ de diámetro). El $\%$ de inhibición se calculó mediante la siguiente formula: $\%$ de inhibición: $100 *$ ( $\Delta$ peso grupo control $-\Delta$ peso tratamiento) / $\Delta$ peso grupo control), (Gonzales 2011, 2007; Seydel 2002).

Componentes de la crema con el sólido A (ácido úsnico), alcohol cetílico 6\%, ácido esteárico $2 \%$, metilparabeno $0,2 \%$, propilparabeno $0,2 \%$ y vaselina sólida $2 \%$. Componentes de la crema base.-Tetranolamina 1\% (TEA), glicerina $6 \%$ y agua destilada C.S.P $100 \%$. 


\section{RESULTADOS Y DISCUSIÓN}

Los resultados de los análisis cualitativo (Marcha fitoquímica y reacciones de coloración) del liquen en estudio, nos indica que contiene fenoles, taninos, ácidos fenólicos, aminoácidos, esteroides, triterpenos, dépsidos, depsidonas, dibenzofuranos, etc.

Elucidación del sólido A, este sólido es soluble en cloroformo y acetona, parcialmente soluble en etanol, e insoluble en agua; tiene un punto de fusión de 203 - $204^{\circ} \mathrm{C}$, tiene un Rf 0,45 idéntico al de una muestra pura de ácido úsnico, en el sistema: tolueno/acetato de etilo/ácido acético glacial 6:4:1v/v/v, se asperjó con una solución de $\mathrm{H}_{2} \mathrm{SO}_{4}$ en $\mathrm{MeOH} 10 \%$; su rotación óptica $[\alpha]^{\mathrm{D}}$ del sólido A, a $25^{\circ} \mathrm{C}$ en cloroformo $(\mathrm{c}=0,52)$ es: +492 . Su tiempo de retención en un análisis cromatográfico por HPLC es de 26,103 min.

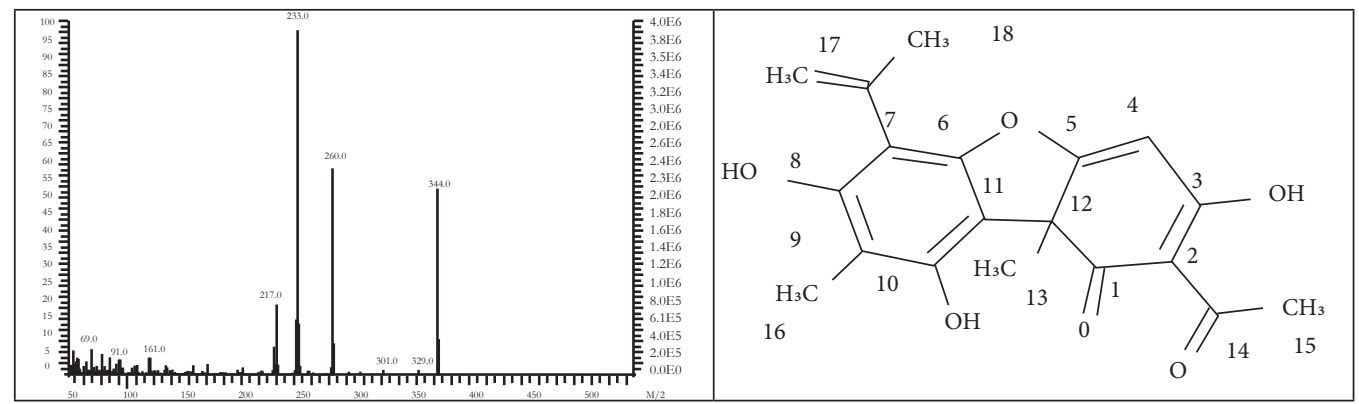

Figura 1. Espectro de masas del sólido A

Figura 2. (+) - Ácido úsnico

El espectro UV-vis, se aprecia los valores de absorbancia máxima (239 y 283 $\mathrm{nm}$ ), los cuales son bastante cercanos comparados con los datos bibliográficos $^{7}$ (234 y 284nm); ambos han sido leídos en cloroformo. Por otro lado, comparando los datos espectroscópicos de IR, RMN-H ${ }^{1}$ y $\mathrm{RMN}-\mathrm{C}^{13}$ con los de la bibliografía (Huneck 1996), se observa una gran similitud; finalmente, analizando el espectro de masa (figura 1), nos indica que se trata del compuesto $\mathrm{C}_{18} \mathrm{H}_{16} \mathrm{O}_{7}$ cuyo peso molecular es 344,0 sus fragmentos son: 260, 233(100), 217, 161. Coincide exactamente con los datos de referencia (Huneck, 1996). Entonces en base a lo anterior proponemos que el sólido A es el (+) - ácido úsnico, cuya estructura química está en la figura 2 .

Para el análisis de metales, el método aplicado para el liquen es una modificación del método la EPA 200.7, Rev. 4.4. EMMC, 1994; en primer lugar se realizó una digestión de la muestra seca, para ello se llevó a cenizas, calentando en la estufa a $600{ }^{\circ} \mathrm{C}$, por 5 horas; luego a las cenizas se atacó con ácidos concentrados (primero con $\mathrm{HCl}$ y luego $\mathrm{HNO}_{3} 10 \mathrm{~mL}$ de cada uno) calentando hasta disolución total, y finalmente se llevó a una solución ácida 500mL. Pos- 
teriormente se llevó al equipo ICP. Los resultados se muestran en la tabla 1. Se observa que los elementos mayoritarios que contiene son: potasio, calcio, fósforo, hierro y magnesio que están entre 4 a 19ppm., todos ellos son importantes para la salud de nuestro cuerpo, y como algunos están en porcentajes altos, como el calcio y potasio, así es recomendable consumirlo.

Tabla 1. Análisis de metales del Liquen everniopsis trulla por ICP

\begin{tabular}{cc}
\hline Metales & $\begin{array}{c}\text { Cantidad encontrada } \\
\text { en } \mathrm{mg} / \mathrm{L}\end{array}$ \\
\hline Potasio & 19,00 \\
Calcio & 15,241 \\
Aluminio & 7,462 \\
Fósforo & 7,217 \\
Hierro & 5,105 \\
Magnesio & 4,15 \\
\hline
\end{tabular}

\begin{tabular}{cc}
\hline Metales & $\begin{array}{c}\text { Cantidad encontrada en } \\
\mathrm{mg} / \mathrm{L}\end{array}$ \\
\hline Sodio & 3,101 \\
Sílice & 2,931 \\
Manganeso & 0,767 \\
Boro & 0,694 \\
Zinc & 0,441 \\
\hline
\end{tabular}

Para la determinación del contenido de fenoles totales, se midió la absorbancia de las soluciones de ácido gálico patrón, ver tabla 2, con estos datos se obtuvo la curva patrón ver figura 3, así determinamos la ecuación para calcular la concentración del ácido gálico; luego, empleando la siguiente fórmula, se relacionó la concentración del ácido gálico con la absorbancia: Acido gálico = (Absorbancia + 0,0771) / 0,0991.

Tabla 2. Absorbancias el estándar de ácido gálico a $760 \mathrm{~nm}$

\begin{tabular}{cc}
\hline Concentración $(\mathrm{mg} / \mathrm{L})$ & $\begin{array}{c}\text { Absorbancia en } \\
\text { promedio }\end{array}$ \\
\hline 1 & 0,0308 \\
2 & 0,1065 \\
3 & 0,2215 \\
4 & 0,3244 \\
5 & 0,4172 \\
\hline
\end{tabular}

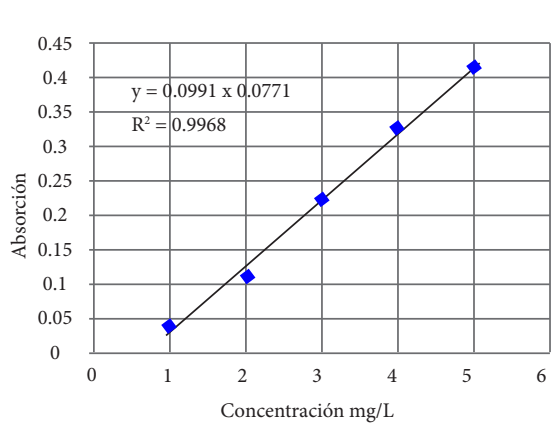

Figura 3. Curva de calibración

Para el análisis del extracto etanólico, se tiene que el peso de la muestra fue de 3,6 mg y su absorbancia medido en promedio fue de 0,2903 entonces la concentración de ácido gálico 3,7073 mg/L ver tabla 3. 
Determinación de la actividad antioxidante, en la tabla 4 se resume las absorbancias promedio y el porcentaje de inhibición de DDPH (Q) dependiendo de la concentración de ácido gálico $(\mathrm{mg} / \mathrm{mL})$. En la tabla 5 , esta los valores para el extracto etanólico. Se observó que la muestra consumió el 50\% de reactivo DPPH a una concentración cercana a $100 \mathrm{mg} / \mathrm{mL}$, mientras que el ácido gálico consumió el 50\% de reactivo DPPH a una concentración aproximada de $9,5 \mathrm{mg} / \mathrm{mL}$.

Tabla 3. Contenido de fenoles totales en la extracto liquénico

\begin{tabular}{ccccc}
\hline Muestra & $\begin{array}{c}\text { Peso } \\
(\mathrm{mg})\end{array}$ & $\begin{array}{c}\text { Absorbancia } \\
\text { promedio }\end{array}$ & $\begin{array}{c}\text { Concentración ácido } \\
\text { gálico }(\mathrm{mg} / \mathrm{L})\end{array}$ & $\begin{array}{c}\text { \% equivalente de ácido } \\
\text { gálico en el extracto }\end{array}$ \\
\hline $\begin{array}{c}\text { Extracto } \\
\text { etanólico }\end{array}$ & 3,6 & 0,2903 & 3,7073 & 20,6 \\
\hline
\end{tabular}

En el estudio farmacológico, hemos empleado la vía tópica, es una vía en la cual se evita la degradación digestiva del principio activo y su metabolismo a nivel hepático. Sin embargo, en la piel concurren una serie de enzimas como las hidrolasas, que también contribuyen a la biotransformación de los fármacos aplicados (Seydel 2002).

Tabla 4. Absorbancias promedio y valor de Q del ácido gálico

\begin{tabular}{ccc}
\hline $\begin{array}{c}\text { Concentración } \\
\text { ácido gálico }(\mathrm{mg} / \\
\mathrm{mL})\end{array}$ & $\begin{array}{c}\text { Absorbancia } \\
\text { promedio }\end{array}$ & $\mathrm{Q}(\%)$ \\
\hline 0 & $0,5012 \pm 0,009$ & 0 \\
0,1 & $0,4133 \pm 0,007$ & 17,5 \\
0,25 & $0,3839 \pm 0,008$ & 23,4 \\
0,5 & $0,3571 \pm 0,005$ & 28,8 \\
1 & $0,3374 \pm 0,007$ & 32,7 \\
5 & $0,2694 \pm 0,001$ & 46,2 \\
10 & $0,2337 \pm 0,003$ & 53,4 \\
30 & $0,1956 \pm 0,005$ & 61,0 \\
45 & $0,1872 \pm 0,001$ & 62,6 \\
\hline
\end{tabular}

Tabla 5. Absorbancia promedio y el valor Q del ácido gálico

\begin{tabular}{ccc}
\hline \multirow{2}{*}{$\begin{array}{c}\text { Concentración } \\
\text { de la muestra en } \\
\text { mg/L }\end{array}$} & \multicolumn{2}{c}{ EXTRACTO M1 } \\
\cline { 2 - 3 } & $\begin{array}{c}\text { Absorbancia } \\
\text { promedio }\end{array}$ & $\mathrm{Q}(\%)$ \\
\hline 0 & 0,6544 & 0 \\
5 & 0,5745 & 12,2 \\
25 & 0,5002 & 23,6 \\
50 & 0,4465 & 31,8 \\
75 & 0,3945 & 39,7 \\
100 & 0,3035 & 53,6 \\
125 & 0,2432 & 62,8 \\
150 & 0,2012 & 69,3 \\
175 & 0,1776 & 72,8 \\
200 & 0,1544 & 76,4 \\
\hline
\end{tabular}


En la tabla 6 se observa el efecto antiinflamatorio del sólido A (ácido úsnico) y del extracto etanólico sobre el edema inducido por xilol en la oreja derecha del ratón, las cremas elaboradas, tuvieron una inhibición 37,27 y 19,54\% respectivamente; la inhibición del diclofenaco mostró una inhibición 32,27\% y el corticoide betametasona $0,05 \%$ tuvo una inhibición significativa $37,27 \%$.

$\%$ de inhibición: $100 *$ ( $\Delta$ peso grupo control $-\Delta$ peso tratamiento $) /(\Delta$ peso grupo control).

Se considera como actividad antiinflamatoria moderada la inhibición del edema del 35 al 65\% y como buen efecto antiinflamatorio un valor mayor de 65\%. En nuestro caso, analizando la tabla 4 vemos que cuando la concentración está alrededor del $10 \mathrm{mg} / \mathrm{mL}$ el \%Q es aproximadamente el 50\%, esto es para el ácido gálico, y para la muestra (extracto etanólico M1), está en 50\% cuando la concentración de la muestra está a $100 \mathrm{mg} / \mathrm{L}$. En la tabla 6 observamos que el efecto anti-inflamatorio del ácido úsnico es equivalente al de betametasona y ligeramente mejor que el diclofenaco.

Tabla 6. Efecto antiinflamatorio sobre el edema inducido por xilol en la oreja derecha del ratón.

\begin{tabular}{cc}
\hline Tratamientos & $\%$ Inhibición edema \\
\hline Control (blanco) & $0,00 \%$ \\
Diclofenaco 1\% & $32,27 \%$ \\
Betametasona 0,05\% & $37,27 \%$ \\
Crema c/ ácido úsnico 0,5\% & $37,72 \%$ \\
Crema c/ extracto liquénico etanólico 1\% & $19,54 \%$ \\
\hline
\end{tabular}

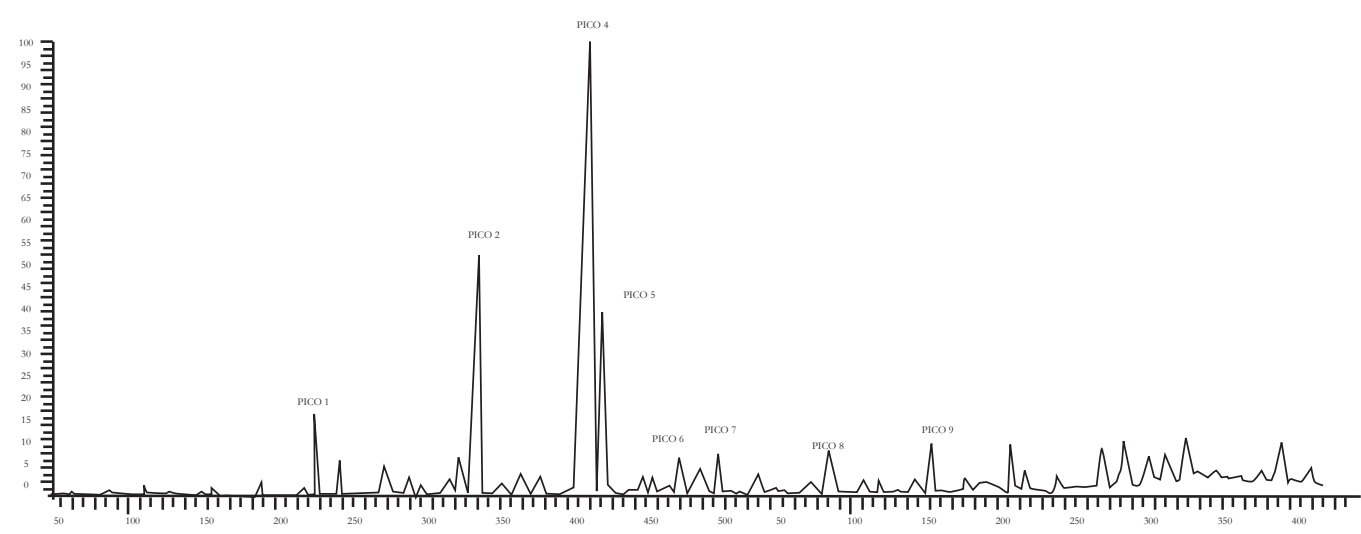

Figura 4. Cromatograma perteneciente a la especie Everniopsis trulla (Ach.) Nyl. 
De los análisis cromatográficos, observando el cromatograma de la figura 4, tenemos una lista de compuestos orgánicos mayoritarios que están en la tabla 7, estos son ácidos grasos, sales orgánicas, alcanos y esteroles; en esta parte sería bueno realizar más investigaciones sobre cada uno de ellos, empleando otros métodos de análisis o mejor dicho diferentes método de tratamiento de la muestra para extraer separadamente a cada tipo de compuestos.

Tabla 7. Compuestos identificados por CG-EM en el extracto de la E. trulla

\begin{tabular}{|c|c|c|c|}
\hline NOMBRE DEL COMPUESTO & $\begin{array}{l}\text { FÓRMULA } \\
\text { MOLECULAR }\end{array}$ & $\begin{array}{l}\text { PESO } \\
\text { MOLECULAR }\end{array}$ & Pico No. \\
\hline metil tetradecanoato & $\mathrm{C}_{15} \mathrm{H}_{30} \mathrm{O}_{2}$ & 242,22 & 1 \\
\hline acido metil éster, (z)-9-hexadecenoico & $\mathrm{C}_{17} \mathrm{H}_{32} \mathrm{O}_{2}$ & 268,24 & 2 \\
\hline acido metil éster hexadecanoico & $\mathrm{C}_{17} \mathrm{H}_{34} \mathrm{O}_{2}$ & 270,26 & 3 \\
\hline 13-metil, $(8 \beta, 13 \beta) 17$-norkau-15-eno & $\mathrm{C}_{20} \mathrm{H}_{32}$ & 272,25 & 4 \\
\hline $\begin{array}{l}\text { acido metil éster }(z, z)-9,12- \\
\text { octadecanoico }\end{array}$ & $\mathrm{C}_{19} \mathrm{H}_{34} \mathrm{O}_{2}$ & 294,26 & 5 \\
\hline acido metil éster (z)-9-octadecenoico & $\mathrm{C}_{19} \mathrm{H}_{36} \mathrm{O}_{2}$ & 296,27 & 6 \\
\hline metil estearato & $\mathrm{C}_{19} \mathrm{H}_{38} \mathrm{O}_{2}$ & 298,29 & 7 \\
\hline acido metil éster araquidónico & $\mathrm{C}_{21} \mathrm{H}_{34} \mathrm{O}_{2}$ & 318,25 & 8 \\
\hline acido metil éster eicosanoico & $\mathrm{C}_{21} \mathrm{H}_{42} \mathrm{O}_{2}$ & 326,32 & 9 \\
\hline acido metil éster docosanoico & $\mathrm{C}_{23} \mathrm{H}_{46} \mathrm{O}_{2}$ & 354,35 & 10 \\
\hline acido metil éster tetracosanoico & $\mathrm{C}_{25} \mathrm{H}_{50} \mathrm{O}_{2}$ & 382,38 & 11 \\
\hline $17 \alpha, 21 \beta, 2 \beta, 30$-bisnorhopano & $\mathrm{C}_{28} \mathrm{H}_{48}$ & 384,38 & 12 \\
\hline tritetracontano & $\mathrm{C}_{43} \mathrm{H}_{88}$ & 604,69 & 13 \\
\hline $17 \alpha, 21 \beta, 2 \beta, 30$-bisnorhopano & $\mathrm{C}_{28} \mathrm{H}_{48}$ & 384,38 & 14 \\
\hline$\gamma$-sitosterol & $\mathrm{C}_{29} \mathrm{H}_{50} \mathrm{O}$ & 414,39 & 15 \\
\hline
\end{tabular}

Los datos pueden presentarse en tablas, ver tabla 1, o en forma de gráficos, ver figura 1. 
Tabla 8. Ácidos grasos del Liquen everniopsis trulla identificados por CG

\begin{tabular}{cccc}
\hline Ácido graso & Fórmula & $\begin{array}{c}\mathrm{t}_{\mathrm{R}} \\
(\mathrm{min})\end{array}$ & $\begin{array}{c}\% \\
\text { relativo }\end{array}$ \\
\hline Ácido laúrico & $\mathrm{C}_{12} \mathrm{H}_{24} \mathrm{O}_{2}$ & 2,77 & 21,13 \\
Ácido palmítico & $\mathrm{C}_{16} \mathrm{H}_{32} \mathrm{O}_{2}$ & 5,55 & 15,46 \\
Ácido esteárico & $\mathrm{C}_{18} \mathrm{H}_{36} \mathrm{O} 2$ & 7,51 & 7,21 \\
Ácido araquídico & $\mathrm{C}_{20} \mathrm{H}_{40} \mathrm{O}_{2}$ & 9,93 & 20,96 \\
Ácido behémico & $\mathrm{C}_{22} \mathrm{H}_{44} \mathrm{O}_{2}$ & 11,32 & 20,96 \\
Ácido lignocérico & $\mathrm{C}_{24} \mathrm{H}_{48} \mathrm{O}_{2}$ & 12,87 & 14,26 \\
\hline
\end{tabular}

Adicionalmente, también se realizó un análisis de ácidos grasos, aplicando un método que comprende 2 etapas, en la primera se realiza la extracción exhaustivamente de los ácidos grasos con $\mathrm{MeOH}-\mathrm{CHCl}_{3} 1: 1$ y la segunda, comprende la saponificación, hidrólisis ácida y metilación de los ácidos grasos seguida del análisis por CG, (Dembistsky, 1992). Este procedimiento se realiza a los estándares y al liquen y finalmente se identifica a los ácidos grasos, los cuales se muestran en la Tabla 8. Revisando los ácidos grasos se observa que tiene algunos ácidos grasos muy importantes para la salud como ácido palmítico esteárico, etc.

\section{CONCLUSIONES}

Se aisló y elucidó al sólido A el cual es el ácido úsnico en base a sus datos espectroscópicos. Se comprobó un efecto antiinflamatorio de las cremas 0,5\% contenido de ácido úsnico aislado del Liquen everniopsis trulla (sólido A), por vía tópica en la oreja derecha del ratón con una inhibición 37,72\%.

El contenido de fenoles totales del extracto del liquen Everniopsis trulla alcanzó ser de $206 \mathrm{mg}$ de ácido gálico/g de extracto.

Se logró determinar la actividad antioxidante en el extracto etanólico del liquen con mayor contenido de compuestos fenólicos, mediante el empleo del reactivo DPPH. Se obtuvo un valor de $\mathrm{CI}_{50}$ a una concentración de $100 \mathrm{mg} / \mathrm{mL}$.

El líquen contiene elementos metálicos muy importantes y en considerable cantidad para la salud, de igual forma contiene ácidos grasos y por su contenido fenólico y antioxidante; es posible recomendar el consumo en ensaladas.

\section{AGRADECIMIENTOS}

Se agradece a la Dra. Magda Chanco, por la identificación botánica; al Dr. J. Elix de la universidad Nacional de Australia por el análisis de HPLC, a la Dra. María 
Caramantín Soriano por los análisis de RMN, al Dr. Arturo San Feliciano por los análisis de espectrometría de masas. Agradecemos a Laboratorio de Análisis y Monitoreo Ambiental, Servicios Analíticos Generales S.A.C. (Lima, Perú) en la persona del Sr. Químico Cesar Poma Pando por permitirnos realizar los análisis CG-EM en sus instalaciones.

\section{REFERENCIAS BIBLIOGRÁFICAS}

Cruzado, M. y otros. (2013). «Determinación de compuestos fenólicos y actividad antioxidante de extractos de alcachofa (Cynara scolymus)». Rev. Soc. Quim. Perú, 19(1), 57-64.

Dembistsky, V. y Johnson, A. 1992. Fatty acid composition of parmelia lichens, Phytochemistry, 31, 841-843.

EPA. 1994, method 2007, Rev. 4.4. EMMC-Version. Determination of metals and trace elements in water and wates bay inductively couple plasma-atomic emission spectrometry-1994.

Gonzales, M. C. y otros. (2011). «Actividad Anti-inflamatoria de extractos y fracciones de Myrcianthes leucoxila, Calea prunifolia en los modelos edema auricular por TPA edema plantar por carragenina y artritis inducida por colágeno». Biosalud, 10(1), 9-18.

Gonzales, M. C. y otros. 2007. «Evaluación de extractos y fracciones de plantas colombianas en modelos de inflamación aguda, subcrónica y crónica». Revista Colombiana Ciencia Química Farm. Vol. 36 (2), 166-174.

Huneck, S. y Yoshimura I. (1996). Identification of lichen substances. Berlin: Springer-Verlag GmbH.

Kika, H. N. y Vilegas, W. (1998). A quimica dos líquenes. Química Nova - Brasil, 21, pp. $110-124$.

Manojlovic, Nedeljko y otros. 2012. «Chemical composition of three parmelia lichens and antioxidant, antimicrobial and cytotoxic activities of some their major metabolites». Phytomedicine 19, 1166-1172.

Seydel, J.K. y Wiese, M. (2002). Drug-membrane interactions. Analysis, drug distribution and modeling. Wiley- $\mathrm{VCH}$.

Presentado: $07 / 02 / 2017$

Aceptado: 15/05/2017

\section{Correspondencia}

Nino Castro Mandujano

ninocastro_2005@yahoo.com 